\title{
118. Das Säge-Treppenschnittverfahren bei der akuten chirurgischen Wundversorgung und bei der Narbenkorrektur
}

C. Walter

Diakoniewerk-Kaiserswerth, Düsseldorf

\section{Sawblade and Stairstep-type Wound Closure in the Treatment of Acute Skin Trauma and in Scar Revisions}

Summary. In order to camouflage a wound or scar, additional incisions are made at an angle to the original wound. These are combined with skin excisions in an attempt to interdigitate the scar line and relieve the tension. The principle of $\mathrm{Z}$-grafting is explained.

Key words: Trauma - Wound closure, sawblade type.

Zusammenfassung. In dem Bemühen, lange Wunden oder Narben weniger sichtbar erscheinen zu lassen, werden quer zur Wunde zusätzliche Incisionen und Hautresektionen vorgenommen. Auf die Technik der Versorgung halbmondförmiger Verletzungen wird eingegangen. Das Prinzip der Z-Plastik wird beschrieben.

Schlïsselwörter: Hautverletzung - Sägeschnittartige Wundversorgung.

\section{Eine neue chirurgische Technik zur funktionsgerechten analen Sphincterersatzplastik im Kindesalter}

\author{
J. Holle, G. Freilinger, B. Mamoli, P. Spängler, F. Braun und R. Krenn \\ II. Chir. Univ. Klinik Wien
}

\begin{abstract}
A New Surgical Technique of Anal Sphincter Reconstruction in Children
Summary. The denervated but vascular musculus gracilis becomes reinnervated by muscular neurotization when transposed to the pelvic floor musculature. At the same time, it adopts all the properties typical of a sphincter muscle. This operation was performed in 5 clinical cases. Follow-up examinations in 3 cases reveal that the reflex contraction and the voluntary contraction of the transposed muscle are exactly similar to those of a sphincter.
\end{abstract}

Key words: Neurotisation, muscular - Surgery, plastic: Anus - Incontinence, anal.

Zusammenfassung. Basierend auf der Tatsache, daß ein denervierter Extremitätenmuskel durch muskuläre Neurotisation über die Beckenbodenmuskulatur reinnerviert und zu einem Tonusmuskel mit den Eigenschaften des Originalsphincters umgestaltet werden kann, wurde an unserer Klinik bisher bei 5 Kindern der Musculus gracilis denerviert und auf die Beckenbodenmuskulatur transportiert. Nachuntersuchungsergebnisse in drei Fällen zeigen die willkürlich wie auch reflektorisch den Sphincteranforderungen angepaßte Funktion.

Schlïsselwörter: Muskuläre Neurotisation - Sphincterplastik - Incontinentia ani. 